\title{
Penta X Syndrome
}

National Cancer Institute

\section{Source}

National Cancer Institute. Penta X Syndrome. NCI Thesaurus. Code C89802.

A rare sex chromosome abnormality in which a female child has 3 extra X chromosomes. 